\title{
Continuous Robust Control for Two-Dimensional Airfoils with Leading- and Trailing-Edge Flaps
}

\author{
Z. Wang* and A. Behalı \\ University of Central Florida, Orlando, Florida 32826 \\ and \\ P. Marzocca \\ Clarkson University, Potsdam, New York 13699 \\ DOI: $\underline{10.2514 / 1.54347}$
}

\begin{abstract}
In this paper, a class of multi-input/multi-output aeroelastic systems with unstructured nonlinear uncertainty is considered. By using leading- and trailing-edge control surface actuations, a continuous robust controller is proposed to suppress the aeroelastic vibrations of a nonlinear wing-section model with plunging and pitching degrees of freedom. Under a mild restriction that the system uncertainties are second-order differentiable, the control design yields a semiglobal asymptotic stability result, leading to rapid suppression of the plunging and pitching motions. Numerical simulation results demonstrate the performance of the multi-input/multi-output continuous robust control toward suppressing aeroelastic vibration and limit cycle oscillations at pre- and postflutter flight-speed regimes, even in the presence of bounded unknown external disturbance.
\end{abstract}

\section{Nomenclature}

$a$

$=$ nondimensional distance from midchord to elastic axis

$b, s=$ semichord and wing-section spans, $\mathrm{m}$

$C_{l \alpha}, C_{m \alpha}=$ rate of change of lift and moment with regard to angle of attack, $1 / \mathrm{rad}$

$C_{m \alpha-\text { eff }}=$ rate of change of effective moment with regard to angle of attack, $1 / \mathrm{rad}$

$C_{l \beta}, C_{m \beta}=$ rate of change of lift and moment with regard to trailing-edge control surface deflections, $1 / \mathrm{rad}$

$C_{m \beta-\text { eff }}=$ rate of change of effective moment with regard to trailing-edge control surface deflections, $1 / \mathrm{rad}$

$C_{l \gamma}, C_{m \gamma}=$ rate of change of lift and moment with regard to leading-edge control surface deflections, $1 / \mathrm{rad}$

$C_{m \gamma-\text { eff }}=$ rate of change of effective moment with regard to leading-edge control surface deflections, $1 / \mathrm{rad}$

$c_{h}=$ structural damping coefficients in plunging, $\mathrm{kg} / \mathrm{s}$

$c_{\alpha}=$ structural damping coefficients in pitching, $\mathrm{kg} \cdot \mathrm{m}^{2} / \mathrm{s}$

$\mathbf{e}_{1}, \mathbf{e}_{2}, \quad=$ tracking error, filtered tracking error, and composite $\mathbf{r}, \mathbf{z}$ error signals

$h \quad=$ plunging displacement, $\mathrm{m}$

$\mathbf{h}, \mathbf{f}, \mathbf{G}_{s}=$ system drift vectors and input gain matrix

$I_{\alpha} \quad=$ inertia of wing section about elastic axis, $\mathrm{kg} \cdot \mathrm{m}^{2}$

$\mathbf{K}, \Gamma, \kappa=$ control gain matrices

$k_{h}=$ structural spring stiffness in plunging, $\mathrm{N} / \mathrm{m}$

$k_{\alpha} \quad=$ structural spring stiffness in pitching, $\mathrm{N} \cdot \mathrm{m}$

$L \quad=$ aerodynamic lift, $\mathrm{N}$

$L_{g} \quad=$ aerodynamic lift due to external disturbance, $\mathrm{N}$

$M \quad=$ aerodynamic moment, $\mathrm{N} \cdot \mathrm{m}$

$M_{g} \quad=$ aerodynamic lift moment due to external disturbance, $\mathrm{N} \cdot \mathrm{m}$

Received 23 March 2011; revision received 29 July 2011; accepted for publication 15 August 2011. Copyright $@ 2011$ by Z. Wang, A. Behal, and P. Marzocca. Published by the American Institute of Aeronautics and Astronautics, Inc., with permission. Copies of this paper may be made for personal or internal use, on condition that the copier pay the $\$ 10.00$ per-copy fee to the Copyright Clearance Center, Inc., 222 Rosewood Drive, Danvers, MA 01923; include the code 0731-5090/12 and $\$ 10.00$ in correspondence with the CCC.

*Graduate Student, Department of Electrical Engineering and Computer Science.

${ }^{\dagger}$ Associate Professor, Mechanical and Aeronautical Engineering.

${ }^{\ddagger}$ Assistant Professor, Department of Electrical Engineering, Computer Science and NanoScience Technology Center.

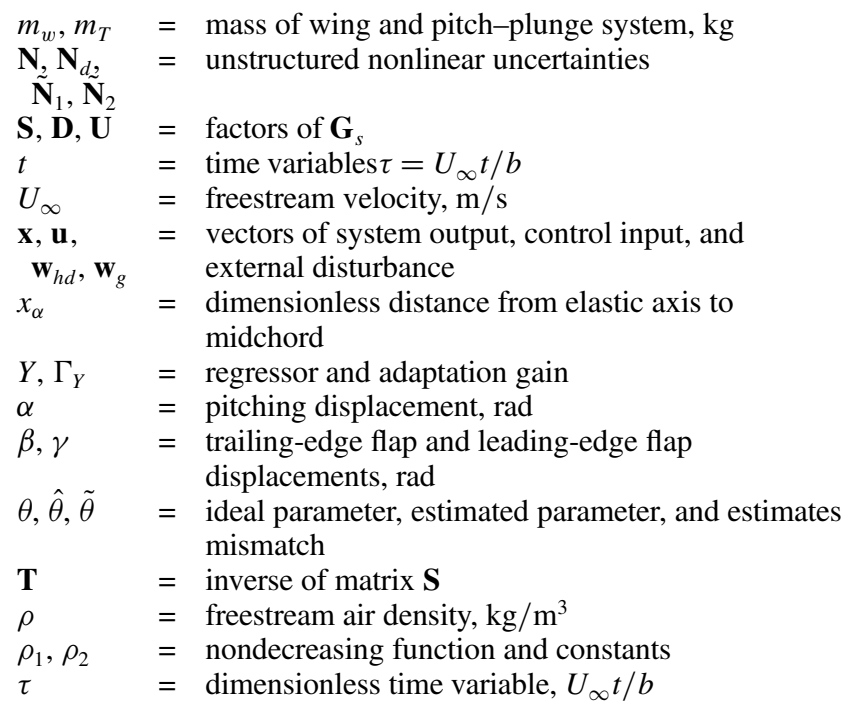

\section{Introduction}

A CTIVE aeroelastic control and flutter suppression of flexible wings have been a fervid topic of investigation by numerous researchers. A number of contributions related to the topic are discussed at length in [1-6]. Among the latest active control methodologies, adaptive and robust control of nonlinear aeroelastic models was presented in [5], the $\mu$-method for robust aeroservoelastic stability analysis in [7], gain scheduled controllers in [8], and neural and adaptive control in [9]. Linear control theory, fee $\overline{d b}$ ack linearizing techniques, and adaptive control strategies have been derived to account for the effect of nonlinear structural stiffness [10]. A model reference variable structure adaptive control system for plunge-displacement and pitch-angle control has been designed using bounds on uncertain functions [11]. This approach yields a high-gain feedback discontinuous control system. In [12-14], an adaptive design method for flutter suppression has been adopted while using measurements of either or both of the pitching and plunging variables. Results in [15] demonstrated that the proposed full state feedback active control mechanism with an estimator was efficient by using a typical section with leading- and trailing-edge flaps. Disturbance rejection, gust alleviation, and flutter suppression were also demonstrated in the experimental investigations.

In [16], an adaptive backstepping design technique was used to control the pitch angle with only output measurements. In [17], an 
adaptive control strategy was proposed using only the feedback for the pitching variable. Its performance toward suppressing flutter and limit cycle oscillations (LCOs), as well as reducing the aeroelastic response in the subcritical flight-speed regime was also demonstrated. Lee and Singh [18] designed a robust control law for the global regulation of a two-degree-of-freedom (2-DOF) aeroelastic system. The model had polynomial type structural nonlinearity and only the pitch angle was measured for feedback. It was also assumed that all the system parameters were unknown to the designer, whereas the bounds of uncertainties were assumed to be known in the control design. Another robust control strategy for active flutter suppression of a nonlinear 2-D wing-flap system was introduced in [19]. An optimized state feedback robust stabilizer with a proportionalintegral observer (PI-observer) was designed in which the PIobserver was adopted to estimate both the system states and the bounds of the nonlinearities in the aeroelastic system. Based on the immersion and invariance approach, the adaptive control design problem for aeroelastic wing sections with structural nonlinearity was solved in [20].

Several control algorithms were proposed in [21-23] for the 2DOF aeroelastic system, which efficiently improved the performance through an extension to a wing section with both trailingedge control surface (TECS) and leading-edge control surface (LECS). An adaptive full state feedback control law was provided in [21]. However, only an inversion of a nominal input gain matrix was used to decouple the control inputs without considering the uncertainty. In [22], adaptive and radial basis function neural network controllers were provided in order to compensate for the system nonlinearity and compared via simulation. In [23], an output feedback adaptive control algorithm was proposed by using a backstepping technique, and an SDU decomposition (symmetricdiagonal-upper triangular factorization) was applied on the input gain matrix to design a singularity-free controller. The backstepping approach in [23] led to a very complicated control design: more than 200 parameters needed to be tuned online, due to significant overparameterization problems. In [24], a modular output feedback controller was proposed to suppress aeroelastic vibrations on unmodeled nonlinear wing section subject to a variety of external disturbances. Although the computation load was reduced greatly in [24] compared with [23], 82 parameters still needed to be updated online for the model-free control algorithm using a neural network approximator.

The active vibration suppression problem for the 2-DOF aeroelastic system with leading- and trailing-edge controls is formulated as an affine-in-the-control multi-input/multi-output (MIMO) system with unknown uncertainty and bounded external disturbance, and numerous progress has been reported in recent years on the control design for this kind of MIMO systems with uncertainty based on a variety of techniques and assumptions. In [25], the highfrequency gain (HFG) matrix $G$ was assumed to be known for the control design. In [26], a control law was proposed that required the existence of a matrix $S$ such that $G S$ is positive-definite and symmetric. Based on the assumption that the HFG matrix was known, an adaptive backstepping technique was proposed for parametric strict feedback systems in [27]. In [28], a Lyapunov-based adaptive output feedback control was designed for a general class of MIMO system with unknown constant parameters, but susceptible to singularities owing to the existence of an algebraic loop in the controller. Later, in [29], this problem was solved by designing a singularity-free output feedback controller with parameter uncertainty. In [30], the proposed controller yields semiglobal uniformly ultimately bounded tracking result while compensating for unstructured uncertainty in both the drift vector and the input matrix. Later, in [31], a locally uniformly ultimately bounded result was obtained by applying an output feedback robust continuous control law for a class of MIMO system with uncertain $\mathcal{C}^{2}$ nonlinearities; a neural network (NN)-based estimator and high-gain observer were used during the control design. Some other examples relating to NN applications in MIMO control can be found in [32]. A summary of the theory and application of robust and sliding mode control in MIMO system can be found in [33].
In this paper, a novel MIMO continuous robust controller (i.e., $\mathcal{C}^{0}$ ) is designed to asymptotically stabilize the MIMO aeroelastic system with unstructured nonlinear uncertainties and bounded unknown external disturbance. The result in this paper is motivated by a singleinput/single-output result presented in [34]. The challenge in extending this result to the MIMO system presented in this paper is due to the coupling of the control inputs, which causes the leadingedge flap displacement to appear as a disturbance term in the closedloop dynamics of the plunging variable. Here, this issue is addressed during the design of the trailing-edge flap displacement via use of robust control alongside a simple adaptive scheme to tackle the $\mathcal{C}^{0}$ component of the coupling-related disturbance terms. The design of the adaptation law is facilitated by the affine-in-the-parameters structure of the uncertainties induced by the control coupling. We note here that adaptation is only carried out for the structured disturbance induced by the control coupling and is not used for the unstructured uncertainty in the system model. Specifically, given the affine-in-the-control MIMO aeroelastic system, the input gain matrix is considered to be unknown, nonsymmetric with nonzero leading principal minors. Based on limited assumptions on the structure of the system nonlinearities and external disturbance, as well as knowledge of the signs of the leading principal minors of the input gain matrix, the problem is solved using an SDU decomposition to facilitate design of singularity-free leading- and trailing-edge robust controllers. Through a Lyapunov analysis, it is possible to show that semiglobal asymptotic stability can be obtained for the tracking errors in the pitching and plunging variables. Simulation results also show that this control strategy can rapidly suppress nonlinear aeroelastic vibrations including flutter and LCOs. Compared with previous work by the authors and others, the proposed control algorithm in this paper significantly reduces the computational burden in the sense that only one parameter needs to be updated during the control implementation. Compared with the uniformly ultimately bounded result obtained in [24] by using the model-free control design with finite control gains, the robust adaptive control design in this paper is able to achieve semiglobal asymptotic stability result with finite control gains, even in the presence of unmodeled external disturbance. We also note that the proposed algorithm requires very little information on the wing-section model; only the signs of leading principal minors of the HFG matrix are needed for the control design.

The rest of this paper is organized as follows. In Sec. II, the system dynamics are introduced. Then, the control objective is defined and the open-loop error system is developed to facilitate the subsequent control design. In Sec. III, the robust feedback control design is proposed followed by a Lyapunov-based analysis of stability of the closed-loop system. Simulation results to confirm the performance and robustness of the controller are presented in Sec. IV, and concluding remarks and future outlook are provided in Sec. $\underline{\mathrm{V}}$.

\section{Aeroelastic Model Configuration and Error System Development}

A wing-section model with 2-DOF in plunging and pitching with both LECS and TECS is illustrated in Fig. 1. The classical aeroelastic governing equations for the sectional wing subject to bounded external disturbance are developed from previous models according to $[\underline{21}, \underline{35}]$

$$
\begin{aligned}
& {\left[\begin{array}{cc}
m_{T} & m_{w} x_{\alpha} b \\
m_{w} x_{\alpha} b & I_{\alpha}
\end{array}\right]\left[\begin{array}{c}
\ddot{h} \\
\ddot{\alpha}
\end{array}\right]+\left[\begin{array}{cc}
c_{h} & 0 \\
0 & c_{\alpha}
\end{array}\right]\left[\begin{array}{c}
\dot{h} \\
\dot{\alpha}
\end{array}\right]+\left[\begin{array}{cc}
k_{h} & 0 \\
0 & k_{\alpha}(\alpha)
\end{array}\right]\left[\begin{array}{l}
h \\
\alpha
\end{array}\right]} \\
& =\left[\begin{array}{c}
-L-L_{g} \\
M+M_{g}
\end{array}\right]
\end{aligned}
$$

All the definitions of symbols used in Eq. (1) can be found in the Nomenclature. The quasi-steady lift $L(\dot{h}, \dot{\alpha}, h, \alpha, \beta, \gamma)$ and aerodynamic moment $M(\dot{h}, \dot{\alpha}, h, \alpha, \beta, \gamma)$ are given by 


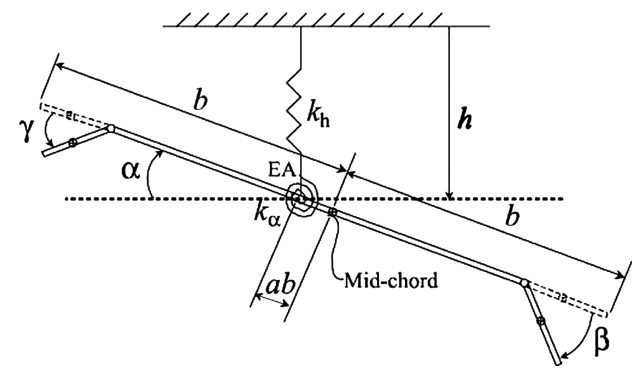

Fig. 1 Two-degree-of-freedom aeroelastic system with both leadingand trailing-edge control surfaces.

$$
\begin{aligned}
L & =\rho U_{\infty}^{2} b s C_{l \alpha}\left(\alpha+\frac{\dot{h}}{U_{\infty}}+\left(\frac{1}{2}-a\right) b \frac{\dot{\alpha}}{U_{\infty}}\right)+\rho U_{\infty}^{2} b s C_{l \beta} \beta \\
& +\rho U_{\infty}^{2} b s C_{l \gamma} \gamma \\
M & =\rho U_{\infty}^{2} b^{2} s C_{m \alpha-\mathrm{eff}}\left(\alpha+\frac{\dot{h}}{U_{\infty}}+\left(\frac{1}{2}-a\right) b \frac{\dot{\alpha}}{U_{\infty}}\right) \\
& +\rho U_{\infty}^{2} b^{2} s C_{m \beta-\mathrm{eff}} \beta+\rho U_{\infty}^{2} b^{2} s C_{m \gamma-\mathrm{eff}} \gamma
\end{aligned}
$$

where $C_{m \alpha-\mathrm{eff}}, C_{m \beta-\mathrm{eff}}$, and $C_{m \gamma-\mathrm{eff}}$ are defined as follows:

$$
\begin{aligned}
& C_{m \alpha-\mathrm{eff}}=\left(\frac{1}{2}+a\right) C_{l \alpha}+2 C_{m \alpha} \\
& C_{m \beta-\mathrm{eff}}=\left(\frac{1}{2}+a\right) C_{l \beta}+2 C_{m \beta} \\
& C_{m \gamma-\mathrm{eff}}=\left(\frac{1}{2}+a\right) C_{l \gamma}+2 C_{m \gamma}
\end{aligned}
$$

Since this paper is mainly focused on the continuous robust control design for 2-D airfoils, the aerodynamic model is limited to linear quasi-steady. The structural nonlinear model accounting for pitch cubic nonlinearities is also a classical one and has been used in many archival literature contributions. The idea was to use a classical nonlinear aeroelastic model, behaving similarly to a Duffing oscillator, to infer about the effect of the control in the postcritical flutter regime, where a nonlinear LCO is experienced by the aeroelastic system. Flutter instability clearly depends upon the type of nonlinearities included in the model. The instabilities exhibited can be rewritten into an input-output representation to facilitate the subsequent control design:

$$
\ddot{\mathbf{x}}=\mathbf{h}(\mathbf{x}, \dot{\mathbf{x}})+\mathbf{w}_{h d}+\mathbf{G}_{s} \mathbf{u}
$$

where $\mathbf{x} \triangleq[h, \alpha]^{T} \in \mathfrak{R}^{2}$ is a vector of system output, $\mathbf{x}$ and $\dot{\mathbf{x}}$ are the state variables, and

$$
\mathbf{u}=\left[u_{1}, u_{2}\right]^{T} \triangleq[\beta, \gamma]^{T} \in \Re^{2}
$$

denotes the control input vector. Here, $\mathbf{h}(\mathbf{x}, \dot{\mathbf{x}}) \in \mathfrak{R}^{2}$ contains unstructured nonlinear uncertainties, $\mathbf{w}_{h d} \in \mathfrak{R}^{2}$ represents the unknown external disturbance terms due to the contributions of the gusts (i.e., $L_{g}$ and $\left.M_{g}\right)$. It is assumed that $\mathbf{h}(\mathbf{x}, \dot{\mathbf{x}})$ is a $\mathcal{C}^{2}$ function and $\mathbf{w}_{h d}, \dot{\mathbf{w}}_{h d}, \ddot{\mathbf{w}}_{h d} \in \mathcal{L}_{\infty}$. Furthermore,

$$
\mathbf{G}_{s} \triangleq\left[\begin{array}{ll}
g_{11} & g_{12} \\
g_{21} & g_{22}
\end{array}\right] \in \mathfrak{R}^{2 \times 2}
$$

is a constant nonsingular input gain matrix explicitly defined as follows:

$$
\begin{aligned}
& g_{11}=-U_{\infty}^{2} \Delta^{-1} \rho b s\left(I_{\alpha} C_{l \beta}+m_{w} x_{\alpha} b^{2} C_{m \beta-\mathrm{eff}}\right) \\
& g_{12}=-U_{\infty}^{2} \Delta^{-1} \rho b s\left(I_{\alpha} C_{l \gamma}+m_{w} x_{\alpha} b^{2} C_{m \gamma-\mathrm{eff}}\right) \\
& g_{21}=U_{\infty}^{2} \Delta^{-1} \rho b s\left(m_{w} x_{\alpha} b C_{l \beta}+m_{T} b C_{m \beta-\mathrm{eff}}\right) \\
& g_{22}=U_{\infty}^{2} \Delta^{-1} \rho b s\left(m_{w} x_{\alpha} b C_{l \gamma}+m_{T} b C_{m \gamma-\mathrm{eff}}\right)
\end{aligned}
$$

where

$$
\Delta \triangleq \operatorname{det}\left(\mathbf{G}_{s}\right)=m_{T} I_{\alpha}-m_{w}^{2} x_{\alpha}^{2} b^{2} \neq 0
$$

Motivated by the matrix decomposition approach introduced in [39] and the facts that both the leading principal minors $g_{11}$ and $\Delta$ are nonzero, $\mathbf{G}_{s}$ can be decomposed as $\mathbf{G}_{s}=\mathbf{S D U}$, where $\mathbf{S}$ is a symmetric positive-definite matrix, $\mathbf{D}$ is a diagonal matrix with diagonal entries +1 or -1 , and $\mathbf{U}$ is an unknown unity uppertriangular matrix. Note that this SDU decomposition is a key factor in the proposed algebraic loop-free controller design. By using the SDU decomposition result from [23], S, D, and $\mathbf{U}$ can be explicitly written as

$$
\begin{gathered}
\mathbf{S}=\left[\begin{array}{cc}
\left|g_{11}\right| & \operatorname{sign}\left(g_{11}\right) g_{21} \\
\operatorname{sign}\left(g_{11}\right) g_{21} & \operatorname{sign}\left(g_{11}\right) \operatorname{sign}(\Delta)\left[g_{22}-g_{11}^{-1} g_{21}\left(g_{12}-g_{21} \operatorname{sign}(\Delta)\right)\right]
\end{array}\right], \quad \mathbf{D}=\left[\begin{array}{cc}
\operatorname{sign}\left(g_{11}\right) & 0 \\
0 & \operatorname{sign}\left(g_{11}\right) \operatorname{sign}(\Delta)
\end{array}\right] \\
\mathbf{U}=\left[\begin{array}{cc}
1 & \frac{\left|g_{11}^{-1}\right|\left(g_{12}-g_{21} \operatorname{sign}(\Delta)\right)}{\operatorname{sign}\left(g_{11}\right)} \\
0 & 1
\end{array}\right]
\end{gathered}
$$

limit cycle oscillations, which might transition into divergent flutter. Cubic hardening effects result in strictly limit cycle oscillations and it is recognized that it is possible to allow the amplitude of the flutter oscillations to grow to a limiting amplitude through the introduction of nonlinear structural stiffness. Dynamic stall and trailing-edge separation using a modified Beddoes-Leishman's formulation in [36-38] will be considered in a future work.

The aerodynamic loads due to the bounded external disturbance can be given as [35]

$$
\begin{gathered}
L_{g}=\rho U_{\infty}^{2} b s C_{l \alpha} w_{G}(\tau) / U_{\infty}=\rho U_{\infty} b s C_{l \alpha} w_{G}(\tau) \\
M_{g}=\left(\frac{1}{2}-a\right) b L_{g}
\end{gathered}
$$

where $w_{G}(\tau)$ denotes the disturbance velocity, and $\tau$ is a dimensionless time variable defined in the Nomenclature. Motivated by the result in $[28,30,31]$, the aeroelastic governing equations (1) where the notation $\operatorname{sign}(\cdot)$ represents the standard signum function. For purposes of control design, we assume that the signs of the leading principal minors of the high-frequency gain matrix $\mathbf{G}_{s}$ are known, i.e., the diagonal matrix $\mathbf{D}$ is assumed to be known. It is to be noted that no knowledge is assumed, during the control design, about the structure of $\mathbf{S}$ and $\mathbf{U}$ other than the fact that they are symmetric positive-definite and unity upper-triangular, respectively. After multiplying both sides of Eq. () with $\mathbf{T} \triangleq \mathbf{S}^{-1} \in \mathfrak{R}^{2 \times 2}$, Eq. (5) can be rewritten as

$$
\mathbf{T} \ddot{\mathbf{x}}=\mathbf{f}(\mathbf{x}, \dot{\mathbf{x}})+\mathbf{w}_{d}+\mathbf{D U} \mathbf{u}
$$

where $\mathbf{T}$ is a symmetric positive-definite matrix, $\mathbf{f}(\mathbf{x}, \dot{\mathbf{x}}) \triangleq$ $\mathbf{T} \cdot \mathbf{h}(\mathbf{x}, \dot{\mathbf{x}}) \in \Re^{2}$, and $\mathbf{w}_{d} \triangleq \mathbf{T} \cdot \mathbf{w}_{h d} \in \mathfrak{R}^{2}$. The tracking error $\mathbf{e}_{1}(t) \in \Re^{2}$ is defined as follows:

$$
\mathbf{e}_{1} \triangleq \mathbf{x}_{d}-\mathbf{x}
$$


where $\mathbf{x}_{d} \in \Re^{2}$ is the desired bounded output vector that is designed to be $\mathcal{C}^{4}$ smooth in deference to the requirements of the subsequent control design such that

$$
\mathbf{x}_{d}^{(i)}(t) \in \mathcal{L}_{\infty} \quad \forall i=1,2,3,4
$$

Since the control objective is to suppress the aeroelastic vibrations, one can simply choose $\mathbf{x}_{d}$ to be zero all the time or use another desirable smooth trajectory $\mathbf{x}_{d}$ along which the actual pitching and plunging variables encoded by $\mathbf{x}$ can be driven toward the origin. Next, an auxiliary error signal $\mathbf{e}_{2}(t) \in \mathfrak{R}^{2}$ and filtered tracking error $\mathbf{r}(t) \in \mathfrak{R}^{2}$ are introduced as follows:

$$
\mathbf{e}_{2}=\dot{\mathbf{e}}_{1}+\mathbf{e}_{1}, \quad \mathbf{r}=\dot{\mathbf{e}}_{2}+\kappa \mathbf{e}_{2}
$$

Then, based on the above definitions, a composite error signal $\mathbf{z}(t) \in \mathfrak{R}^{6}$ can be defined as follows:

$$
\mathbf{z} \triangleq\left[\mathbf{e}_{1}^{T}, \mathbf{e}_{2}^{T}, \mathbf{r}^{T}\right]^{T}
$$

By taking the time derivative of $\mathbf{r}$ and substituting from the derivative of $\mathbf{e}_{2}$, one can easily obtain the following relation:

$$
\dot{\mathbf{r}}=\ddot{\mathbf{e}}_{2}+\kappa \dot{\mathbf{e}}_{2}
$$

After premultiplying both sides of Eq. (13) by $\mathbf{T}$ and applying the

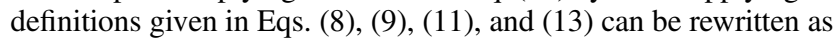

$$
\mathbf{T} \dot{\mathbf{r}}=\mathbf{T}\left(\dddot{\mathbf{x}}_{d}+\ddot{\mathbf{e}}_{1}+\kappa \dot{\mathbf{e}}_{2}\right)-\dot{\mathbf{f}}(\mathbf{x}, \dot{\mathbf{x}})+\mathbf{e}_{2}-\dot{\mathbf{w}}_{d}-\mathbf{D U} \dot{\mathbf{u}}-\mathbf{e}_{2}
$$

Furthermore, given a strictly upper-triangular matrix $\overline{\mathbf{U}}=\mathbf{D}-$ DU $\in \Re^{2 \times 2}$, the open-loop dynamics of Eq. (14) can be rewritten as follows:

$$
\mathbf{T} \dot{\mathbf{r}}=\mathbf{T}\left(\dddot{\mathbf{x}}_{d}+\ddot{\mathbf{e}}_{1}+\kappa \dot{\mathbf{e}}_{2}\right)-\dot{\mathbf{f}}(\mathbf{x}, \dot{\mathbf{x}})+\mathbf{e}_{2}-\dot{\mathbf{w}}_{d}+\overline{\mathbf{U}} \dot{\mathbf{u}}-\mathbf{D} \dot{\mathbf{u}}-\mathbf{e}_{2}
$$

To facilitate the full state control design for the above open-loop dynamics, the following manipulations needs to be applied to Eq. (15):

$$
\mathbf{T} \dot{\mathbf{r}}=\mathbf{N}+\overline{\mathbf{U}} \dot{\mathbf{u}}-\mathbf{D} \dot{\mathbf{u}}-\mathbf{e}_{\mathbf{2}}
$$

where $\overline{\mathbf{U}} \dot{\mathbf{u}} \in \Re^{2}$ can be explicitly expressed as

$$
\overline{\mathbf{U}} \dot{\mathbf{u}}=\left[\begin{array}{ll}
\bar{U}_{12} \dot{u}_{2} & 0
\end{array}\right]^{T}
$$

and

$$
\mathbf{N}\left(\mathbf{x}, \dot{\mathbf{x}}, \ddot{\mathbf{x}}, \mathbf{x}_{\mathbf{d}}, \dot{\mathbf{x}}_{\mathbf{d}}, \ddot{\mathbf{x}}_{\mathbf{d}}, \dddot{\mathbf{x}}_{\mathbf{d}}, \dot{\mathbf{w}}_{\mathbf{d}}\right) \in \Re^{2}
$$

in Eq. (16) is defined as

$$
\mathbf{N}=\mathbf{T}\left(\dddot{\mathbf{x}}_{d}+\ddot{\mathbf{e}}_{1}+\kappa \dot{\mathbf{e}}_{2}\right)-\dot{\mathbf{f}}(\mathbf{x}, \dot{\mathbf{x}})+\mathbf{e}_{2}-\dot{\mathbf{w}}_{d}=\mathbf{N}_{d}+\tilde{\mathbf{N}}_{1}
$$

where

$$
\mathbf{N}_{d}=\mathbf{N}\left(\mathbf{x}_{d}, \dot{\mathbf{x}}_{d}, \ddot{\mathbf{x}}_{d}, \dddot{\mathbf{x}}_{d}, \dot{\mathbf{w}}_{d}\right) \in \mathfrak{R}^{2}, \quad \tilde{\mathbf{N}}_{1}=\mathbf{N}-\mathbf{N}_{d} \in \Re^{2} \mu
$$

One can also demonstrate that $\mathbf{N}_{d}, \dot{\mathbf{N}}_{d} \in \mathcal{L}_{\infty}$ given the boundedness properties of $\mathbf{x}_{d}$ and $\mathbf{w}_{d}$ and the $\mathcal{C}^{2}$ condition on $\mathbf{f}(\mathbf{x}, \dot{\mathbf{x}})$. Furthermore, by using the fact that $\mathbf{N}$ is continuously differentiable, one can easily get the following result:

$$
\left\|\tilde{\mathbf{N}}_{1}\right\| \leq \rho_{1}(\|\mathbf{z}\|)\|\mathbf{z}\|
$$

where $\rho_{1}(\cdot)$ is a global invertible nondecreasing function.

\section{State Feedback Control Development}

\section{A. Full State Feedback Control Design}

In this section, it is assumed that both the output vector $\mathbf{x}$ and their first-order time derivative $\dot{\mathbf{x}}$ can be measured directly. Based on the previous assumptions, the following full state feedback control law is proposed:

$$
\begin{aligned}
\mathbf{u}(t) & =\mathbf{D}^{-1}\left\{(\mathbf{K}+\mathbf{I}) \mathbf{e}_{2}(t)-(\mathbf{K}+\mathbf{I}) \mathbf{e}_{2}(0)+\int_{0}^{t}\left[\left[\begin{array}{ll}
Y \hat{\theta} & 0
\end{array}\right]^{T}\right.\right. \\
& \left.\left.+(\mathbf{K}+\mathbf{I}) \kappa \mathbf{e}_{2}(\tau)+\Gamma \operatorname{sign}\left(\mathbf{e}_{2}(\tau)\right)\right] \mathrm{d} \tau\right\}
\end{aligned}
$$

where

$$
\mathbf{K} \triangleq \mathbf{K}_{p}+\operatorname{diag}\left\{K_{d 1}, 0\right\} \in \mathfrak{R}^{2 \times 2}, \quad \Gamma \in \mathfrak{R}^{2 \times 2}
$$

are both diagonal gain matrices, and $\mathbf{I} \in \mathfrak{R}^{2 \times 2}$ is an identity matrix and $\kappa$ is a positive constant. By taking the time derivative of Eq. (20), one can obtain

$$
\dot{\mathbf{u}}(t)=\mathbf{D}^{-1}\left\{\left[\begin{array}{ll}
Y \hat{\theta} & 0
\end{array}\right]^{T}+(\mathbf{K}+\mathbf{I}) \mathbf{r}(t)+\Gamma \operatorname{sign}\left(\mathbf{e}_{2}(t)\right)\right\}
$$

where $Y \in \Re$ and $\hat{\theta}(t) \in \Re$ denote a regressor and a parameter estimate, which will be defined subsequently. After substituting Eq. (21) into the open-loop dynamics (16), the following closed-loop dynamics are obtained:

$$
\mathbf{T} \dot{\mathbf{r}}=\mathbf{N}_{d}+\tilde{\mathbf{N}}_{1}+\overline{\mathbf{U}} \dot{\mathbf{u}}-\left[\begin{array}{ll}
Y \hat{\theta} & 0
\end{array}\right]^{T}-(\mathbf{K}+\mathbf{I}) \mathbf{r}-\Gamma \operatorname{sign}\left(\mathbf{e}_{2}\right)-\mathbf{e}_{2}
$$

where $\mathbf{N}_{d}$ and $\tilde{\mathbf{N}}_{1}$ have been previously defined in Eq. (18). Based on Eq. (21), one can rewrite $\overline{\mathbf{U}} \dot{\mathbf{u}}$ in Eq. (22) as

$$
\overline{\mathbf{U}} \dot{\mathbf{u}}=\left[\begin{array}{ll}
Y \theta & 0
\end{array}\right]^{T}+\tilde{\mathbf{N}}_{2}
$$

where $Y \triangleq D_{22}^{-1} \Gamma_{22} \operatorname{sign}\left(e_{22}\right)$ is the regressor, and $\theta \triangleq \bar{U}_{12}$ is the unknown upper-right element from the strictly upper-triangular matrix $\overline{\mathbf{U}}$. Here, $D_{i i}$ and $\Gamma_{i i}$ denote the $i$ th diagonal element in the matrices $\mathbf{D}$ and $\Gamma, e_{2 i}$ represents the $i$ th element in auxiliary error signal $\mathbf{e}_{2}$. Furthermore, in Eq. (23),

$$
\tilde{\mathbf{N}}_{2} \triangleq\left[\bar{U}_{12} D_{22}^{-1}\left(K_{22}+1\right) r_{2} \quad 0\right]^{T} \in \Re^{2}
$$

can be upperbounded as $\left\|\tilde{\mathbf{N}}_{2}\right\| \leq \rho_{2}\|\mathbf{z}\|$, where $\rho_{2}$ is a unknown constant that depends on the control gain $K_{22}$. Also note that $K_{i i}$ denotes the $i$ th diagonal element in the control gain matrix $\mathbf{K}$. After substituting Eq. (23) into Eq. (22), the closed-loop dynamics can be further expressed as

$$
\mathbf{T} \dot{\mathbf{r}}=\mathbf{N}_{d}+\tilde{\mathbf{N}}_{1}+\tilde{\mathbf{N}}_{2}+\left[\begin{array}{ll}
Y \tilde{\theta} & 0
\end{array}\right]^{T}-(\mathbf{K}+\mathbf{I}) \mathbf{r}-\Gamma \operatorname{sign}\left(\mathbf{e}_{2}\right)-\mathbf{e}_{2}
$$

where $\tilde{\theta} \triangleq \theta-\hat{\theta}$ is a parameter estimation error. Motivated by the ensuing stability analysis, the adaptation law for $\hat{\theta}$ is given as follows:

$$
\dot{\hat{\theta}}=\Gamma_{Y} Y r_{1}
$$

where $r_{i}$ is the $i$ th element in the filtered error signal $\mathbf{r}, Y$ has been defined above, and $\Gamma_{Y} \in \mathfrak{R}$ is a positive adaptation gain. It is important to note that $r_{1}$ is unmeasurable, since it consists of $\dot{\mathbf{e}}_{2}$, which depends on state variable $\ddot{\mathbf{x}}$. Therefore, the aforementioned adaptation law for $\hat{\theta}$ cannot be implemented directly in the full state feedback control design. However, integration by parts can be used to obtain the following measurable expression for $\hat{\theta}(t)$ :

$$
\begin{gathered}
\hat{\theta}(t)=\Gamma_{Y} D_{22}^{-1} \Gamma_{22}\left\{\sum_{j=1}^{n}\left[e_{21}\left(t_{j, f}^{+}\right)-e_{21}\left(t_{j, 0}^{+}\right)\right]-\sum_{k=1}^{m}\left[e_{21}\left(t_{k, f}^{-}\right)\right.\right. \\
\left.\left.-e_{21}\left(t_{k, 0}^{-}\right)\right]\right\}+\int_{0}^{t} \Gamma_{Y} Y \kappa e_{21}(\tau) \mathrm{d} \tau \\
\operatorname{sign}\left(e_{22}\right)=\left\{\begin{array}{lll}
1, & \forall t \in\left(t_{j, 0}^{+}, t_{j, f}^{+}\right), & j=1, \ldots, n \\
-1, & \forall t \in\left(t_{k, 0}^{-}, t_{k, f}^{-}\right), & k=1, \ldots, m \\
0, & \text { otherwise }
\end{array}\right.
\end{gathered}
$$


where

$$
\begin{aligned}
& (0, t]=\left(t_{1,0}^{+}, t_{1, f}^{+}\right] \cup \cdots \cup\left(t_{n, 0}^{+}, t_{n, f}^{+}\right] \cup\left(t_{1,0}^{-}, t_{1, f}^{-}\right] \\
& \quad \times \cup \cdots \cup\left(t_{m, 0}^{-}, t_{m, f}^{-}\right] .\left(t_{j, 0}^{+}, t_{j, f}^{+}\right), \quad j=1, \ldots, n
\end{aligned}
$$

represents all the interval when $e_{22}>0$, and $\left(t_{k, 0}^{-}, t_{k, f}^{-}\right)(k=1, \ldots, m)$ represents all the interval when $e_{22}<0$. Here we have taken advantage of the fact that $e_{22}$ are measurable.

\section{B. Stability Analysis}

To facilitate the stability analysis under the full state feedback control design, the following lemma will be used.

Lemma 1: For the following auxiliary function $Q(t) \in \Re$,

$$
Q=\mathbf{r}^{T}\left(\mathbf{N}_{d}-\Gamma \operatorname{sign}\left(\mathbf{e}_{2}\right)\right)
$$

if the control gain matrix $\Gamma$ is chosen as

$$
\Gamma_{i, i}>\left\|N_{d, i}\right\|_{\mathcal{L} \infty}+\frac{1}{\kappa}\left\|\dot{N}_{d, i}\right\|_{\mathcal{L} \infty}, \quad \forall i=1,2
$$

where $N_{d, i}$ is the $i$ th element in the vector $\mathbf{N}_{d}$, the following integral relation holds valid:

$$
\int_{0}^{t} Q(\tau) \mathrm{d} \tau \leq \varsigma_{Q}
$$

where

$$
\varsigma_{Q}=\sum_{i=1}^{2}\left[\Gamma_{i, i}\left|e_{2, i}(0)\right|-e_{2, i}(0) N_{d, i}(0)\right]
$$

Proof of this lemma can be found in [34].

Theorem 1: Provided the diagonal entries of the control gain matrix $\mathbf{K}$ defined in Eq. (20) is chosen to be large enough relative to the size of the initial conditions, $\Gamma$ is chosen according to Eq. (28), and $\kappa>1 / 2$, the proposed continuous robust control design ensures semiglobal asymptotic stability in the sense that the error signals $\mathbf{e}_{1}, \dot{\mathbf{e}}_{1}(t), \ddot{\mathbf{e}}_{1}(t) \rightarrow 0$ as $t \rightarrow \infty$.

Proof: First, a nonnegative Lyapunov function candidate $V_{0}$ is defined as

$$
V_{0}=\frac{1}{2} \sum_{i=1}^{2} \mathbf{e}_{i}^{T} \mathbf{e}_{i}+\frac{1}{2} \mathbf{r}^{T} \mathbf{T r}+\frac{1}{2} \tilde{\theta}^{T} \Gamma_{Y}^{-1} \tilde{\theta}+P
$$

where the nonnegative auxiliary function $P$ can be defined as follows:

$$
P=\varsigma_{Q}-\int_{0}^{t} Q(\tau) \mathrm{d} \tau
$$

and Eq. ( $\underline{30})$ can be bounded as

$$
\lambda_{1}\|\mathbf{y}\|^{2} \leq V_{0} \leq \lambda_{2}\|\mathbf{y}\|^{2}
$$

where

$$
\begin{gathered}
\mathbf{y}=\left[\begin{array}{ccc}
\mathbf{z} & \tilde{\theta} & \sqrt{P}
\end{array}\right]^{T} \in \mathfrak{R}^{8}, \quad \lambda_{1}=\frac{1}{2} \min \left\{1, \underline{T}, \Gamma_{Y}^{-1}\right\} \\
\lambda_{2}=\frac{1}{2} \max \left\{2, \bar{T}, \Gamma_{Y}^{-1}\right\}
\end{gathered}
$$

and $\underline{T}$ and $\bar{T}$ represent the minimum and maximum eigenvalues of $\mathbf{T}$. Upon taking the time derivative of Eq. (30), we obtain

$$
\dot{V}_{0}=\mathbf{e}_{1}^{T} \dot{\mathbf{e}}_{1}+\mathbf{e}_{2}^{T} \dot{\mathbf{e}}_{2}+\mathbf{r}^{T} \mathbf{T} \dot{\mathbf{r}}+\tilde{\theta} \Gamma_{Y}^{-1} \dot{\tilde{\theta}}-Q
$$

Then, by substituting from Eqs. (11), (24), (25), and (27), the above expression can be rewritten as

$$
\begin{aligned}
\dot{V}_{0} & =\mathbf{e}_{1}^{T}\left(\mathbf{e}_{2}-\mathbf{e}_{1}\right)+\mathbf{e}_{2}^{T}\left(\mathbf{r}-\kappa \mathbf{e}_{2}\right)-\tilde{\theta} \Gamma_{Y}^{-1} \Gamma_{Y} Y r_{1}-\mathbf{r}^{T}\left(\mathbf{N}_{d}\right. \\
& \left.-\Gamma \operatorname{sign}\left(\mathbf{e}_{2}\right)\right)+\mathbf{r}^{T}\left[\mathbf{N}_{d}+\tilde{\mathbf{N}}_{1}+\tilde{\mathbf{N}}_{2}+\left[\begin{array}{ll}
Y \tilde{\theta} & 0
\end{array}\right]^{T}\right. \\
& \left.-(\mathbf{K}+\mathbf{I}) \mathbf{r}-\Gamma \operatorname{sign}\left(\mathbf{e}_{2}\right)-\mathbf{e}_{2}\right]
\end{aligned}
$$

After canceling matching terms, one obtains

$$
\dot{V}_{0}=\mathbf{e}_{1}^{T} \mathbf{e}_{2}-\mathbf{e}_{1}^{T} \mathbf{e}_{1}-\kappa \mathbf{e}_{2}^{T} \mathbf{e}_{2}+\mathbf{r}^{T} \tilde{\mathbf{N}}_{1}+\mathbf{r}^{T} \tilde{\mathbf{N}}_{2}-\mathbf{r}^{T}(\mathbf{K}+\mathbf{I}) \mathbf{r}
$$

By using the fact that $\mathbf{e}_{1}^{T} \mathbf{e}_{2} \leq \frac{1}{2} \mathbf{e}_{1}^{T} \mathbf{e}_{1}+\frac{1}{2} \mathbf{e}_{2}^{T} \mathbf{e}_{2}$, an upper bound for Eq. (34) can be expressed as

$$
\begin{aligned}
\dot{V}_{0} & \leq-\frac{1}{2}\left\|\mathbf{e}_{1}\right\|^{2}-\left(\kappa-\frac{1}{2}\right)\left\|\mathbf{e}_{2}\right\|^{2}-\|\mathbf{r}\|^{2}+\|\mathbf{r}\|\left\|\tilde{\mathbf{N}}_{1}\right\| \\
& +\|\mathbf{r}\|\left\|\tilde{\mathbf{N}}_{2}\right\|-\lambda_{K}\|\mathbf{r}\|^{2}-K_{d 1} r_{1}^{2}
\end{aligned}
$$

where $\kappa>1 / 2$ and $\lambda_{K}$ is the maximum eigenvalue for the gain matrix $\mathbf{K}_{p} . r_{1}$ denotes the first element in $\mathbf{r} \in \mathfrak{R}^{2}$. Thus, $\dot{V}_{0}$ can be further upperbounded as

$$
\dot{V}_{0} \leq-\lambda_{3}\|\mathbf{z}\|^{2}+\rho_{1}(\|\mathbf{z}\|)\|\mathbf{r}\|\|\mathbf{z}\|-\lambda_{K}\|\mathbf{r}\|^{2}-K_{d 1} r_{1}^{2}+r_{1} \rho_{2}\|\mathbf{z}\|
$$

where $\lambda_{3}=\min \{1 / 2,(\kappa-1 / 2)\}$. Then, by adding and subtracting terms

$$
\frac{\rho_{2}^{2}}{4 K_{d 1}}\|\mathbf{z}\|^{2}, \quad \frac{\rho_{1}^{2}(\|\mathbf{z}\|)}{4 \lambda_{K}}\|\mathbf{z}\|^{2}
$$

from the right-hand side of the above inequality, one can upperbound $\dot{V}_{0}$ as follows:

$$
\begin{aligned}
\dot{V}_{0} & \leq-\lambda_{4}\|\mathbf{z}\|^{2}-\left(\frac{\lambda_{3}-\lambda_{4}}{2}-\frac{\rho_{1}^{2}(\|\mathbf{z}\|)}{4 \lambda_{K}}\right)\|\mathbf{z}\|^{2} \\
& -\left(\frac{\lambda_{3}-\lambda_{4}}{2}-\frac{\rho_{2}^{2}}{4 K_{d 1}}\right)\|\mathbf{z}\|^{2}
\end{aligned}
$$

Given a positive constant $\lambda_{4}<\lambda_{3}$, one can first choose $\mathbf{K}_{p}$ such that

$$
\lambda_{K}>\frac{\rho_{1}^{2}(\|\mathbf{z}\|)}{2\left(\lambda_{3}-\lambda_{4}\right)}
$$

or, equivalently,

$$
\mathbf{z}(t) \in \mathcal{D}_{\rho} \triangleq\left\{\mathbf{z} \mid\|\mathbf{z}\|<\rho_{1}^{-1}\left(\sqrt{2 \lambda_{K}\left(\lambda_{3}-\lambda_{4}\right)}\right)\right\}
$$

This ensures that the first parenthesized term in Eq. (37) is nonnegative. Motivated by Theorem 8.4 in [40] and the definition of $\mathbf{y}$ and $\mathcal{D}_{\rho}$, the region $\mathcal{D}$ can be explicitly given as

$$
\mathcal{D} \triangleq\left\{\mathbf{y} \mid\|\mathbf{y}\|<\rho_{1}^{-1}\left(\sqrt{2 \lambda_{K}\left(\lambda_{3}-\lambda_{4}\right)}\right)\right\}
$$

Since $\mathbf{K} \triangleq \mathbf{K}_{p}+\operatorname{diag}\left\{K_{d 1}, 0\right\}$, it is clear to see that $K_{22}$ is determined only by $\mathbf{K}_{p}$ and is independent of $K_{d 1}$. Based on the fact that $\rho_{2}$ depends on $K_{22}$, one can design

$$
K_{d 1}>\frac{\rho_{2}^{2}}{2\left(\lambda_{3}-\lambda_{4}\right)}
$$

such that the second parenthesized term in Eq. (37) is nonnegative. Thus, it is straightforward to prove that

$$
\dot{V}_{0} \leq-\lambda_{4}\|\mathbf{z}\|^{2}=-W(\mathbf{y}), \quad \forall \mathbf{y} \in \mathcal{D}
$$

From Eqs. (30) and (38), it is known that $V_{0} \in \mathcal{L}_{\infty}$, and it is also straightforward to see that $\mathbf{e}_{1}, \mathbf{e}_{2}, \mathbf{r}, \tilde{\theta} \in \mathcal{L}_{\infty}$ and $\dot{\mathbf{e}}_{1}, \hat{\theta} \in \mathcal{L}_{\infty}$ given Eq. (11). Then, by using (11), one can easily see that $\dot{\mathbf{e}}_{2} \in \mathcal{L}_{\infty}$, which further implies that $\ddot{\mathbf{e}}_{1} \in \overline{\mathcal{L}_{\infty}}$. Next, given the fact that $\mathbf{x}_{d}$ is $\mathcal{C}^{4}$ smooth and $\mathbf{e}_{1}, \dot{\mathbf{e}}_{1}, \ddot{\mathbf{e}}_{1} \in \mathcal{L}_{\infty}$, it is possible to show that $\mathbf{x}, \dot{\mathbf{x}}, \ddot{\mathbf{x}} \in \mathcal{L}_{\infty}$ by using the definition in Eq. (9) and $\mathbf{f}(\mathbf{x}, \dot{\mathbf{x}}) \in \mathcal{L}_{\infty}$. Now, by using Eq. (ㅁ) and 
Table 1 Wing-section parameters

\begin{tabular}{cc}
\hline \hline$a=-0.6719$ & $I_{c g w}=0.04342 \mathrm{~kg} \cdot \mathrm{m}^{2}$ \\
$b=0.1905 \mathrm{~m}$ & $I_{\alpha}=I_{c a m}+I_{c g w}+m_{\text {wing }} r_{c g}^{2} \mathrm{~kg} \cdot \mathrm{m}^{2}$ \\
$C_{l \alpha}=6.757 \mathrm{rad}^{-1}$ & $k_{h}=2844 \mathrm{~N} / \mathrm{m}$ \\
$C_{l \beta}=3.774 \mathrm{rad}^{-1}$ & $k_{\alpha}(\alpha)=12.77+53.47 \alpha+1003 \alpha^{2} \mathrm{~N} \cdot \mathrm{m}$ \\
$C_{l \gamma}=-0.1566 \mathrm{rad}^{-1}$ & $m_{T}=15.57 \mathrm{~kg}$ \\
$C_{m \alpha}=0 \mathrm{rad}^{-1}$ & $m_{w}=5.23 \mathrm{~kg}$ \\
$C_{m \beta}=-0.6719 \mathrm{rad}^{-1}$ & $m_{\text {wing }}=4.340 \mathrm{~kg}$ \\
$C_{m \gamma}=-0.1005 \mathrm{rad}^{-1}$ & $r_{c g}=-b(0.0998+a) \mathrm{m}$ \\
$c_{h}=27.43 \mathrm{~kg} / \mathrm{s}$ & $s=0.5945 \mathrm{~m}$ \\
$c_{\alpha}=0.0360 \mathrm{~N} \cdot \mathrm{s}$ & $x_{a}=r_{c g} / b$ \\
$I_{\text {cam }}=0.04697 \mathrm{~kg} \cdot \mathrm{m}^{2}$ & $\rho=1.225 \mathrm{~kg} \cdot \mathrm{m}^{3}$ \\
\hline
\end{tabular}

the fact that $\mathbf{w}_{d} \in \mathcal{L}_{\infty}$, one can show that $\mathbf{u} \in \mathcal{L}_{\infty}$. Based on $\mathbf{r} \in \mathcal{L}_{\infty}$, we can see that $\dot{u}_{2} \in \mathcal{L}_{\infty}$ according to Eq. (21). Based on the definition $Y=D_{22}^{-1} \Gamma_{22} \operatorname{sign}\left(e_{22}\right)$ and previous boundedness result on $\hat{\theta}$, one can also prove that $\dot{u}_{1} \in \mathcal{L}_{\infty}$ given the definition in Eq. (21), which further implies $\dot{\mathbf{r}} \in \mathcal{L}_{\infty}$ by using the definition in Eq. (15). Based on the above analysis, one can draw the conclusion that $\dot{W}=-\lambda_{4} \mathbf{z}^{T} \dot{\mathbf{z}} \in \mathcal{L}_{\infty}$ and $W(\mathbf{y})$ is uniformly continuous. Based on the definition of $\mathcal{D}$, one can also define region $\mathcal{S}$ as

$$
\mathcal{S} \triangleq\left\{\mathbf{y} \in \mathcal{D} \mid W_{2}(\mathbf{y})<\lambda_{1}\left(\rho_{1}^{-1}\left(\sqrt{2 \lambda_{K}\left(\lambda_{3}-\lambda_{4}\right)}\right)\right)^{2}\right\}
$$

Now, one can use Theorem 8.4 in [40] to prove $\|\mathbf{z}\| \rightarrow 0$ as $t \rightarrow \infty \forall \mathbf{y}(0) \in \mathcal{S}$. From Eq. (12), one can see that $\mathbf{e}_{1}(t), \mathbf{e}_{2}(t), \mathbf{r}(t) \rightarrow 0$ as $t \rightarrow \infty$. Note that $\mathbf{e}_{2}(t), \mathbf{r}(t) \rightarrow 0$ as $t \rightarrow$ $\infty$ further implies that $\dot{\mathbf{e}}_{1}(t), \ddot{\mathbf{e}}_{1}(t) \rightarrow 0$, as $t \rightarrow \infty$ by using Eq. (11). Also note that region of attraction $\mathcal{S}$ in this problem can be made arbitrarily large to include any initial condition through choosing a large enough control gain. The above facts imply that our stability result is semiglobal.

Remark 1: When only measurements available are the pitching and plunging displacements, the remaining states are estimated through the use of a high-gain observer (HGO). When $\mathbf{x}(t)$ is the output of the system and the only measurable state vector, the sole measurable error signal is $\mathbf{e}_{1}(t)$ given the knowledge of $\mathbf{x}(t)$ and $\mathbf{x}_{d}(t)$. Motivated by the result in [40], an estimate for the error signals $\hat{\mathbf{e}}_{1}$ and $\hat{\mathbf{e}}_{2}$ can be obtained via the following HGO:

$$
\begin{aligned}
& \dot{\hat{\mathbf{e}}}_{1}=\hat{\mathbf{e}}_{2}-\hat{\mathbf{e}}_{1}+\frac{\alpha_{1}}{\epsilon}\left(\mathbf{e}_{1}-\hat{\mathbf{e}}_{1}\right) \\
& \dot{\hat{\mathbf{e}}}_{2}=\hat{\mathbf{r}}-\kappa \hat{\mathbf{e}}_{2}+\frac{\alpha_{2}}{\epsilon^{2}}\left(\mathbf{e}_{1}-\hat{\mathbf{e}}_{1}\right) \\
& \dot{\hat{\mathbf{r}}}=\frac{\alpha_{3}}{\epsilon^{3}}\left(\mathbf{e}_{1}-\hat{\mathbf{e}}_{1}\right)
\end{aligned}
$$

where $\alpha_{i} \in \Re \forall i=1,2,3$ are gain constants and $\epsilon$ is a small positive constant. Note that in this paper, only $\hat{\mathbf{e}}_{1}$ and $\hat{\mathbf{e}}_{2}$ are used in the output feedback control design. To suppress the peaking phenomenon due to using HGO, we modify the full state control design in Eq. (20) to an output feedback saturated controller as $\mathbf{u}_{s}(t)=\operatorname{sat}\left\{\mathbf{u}\left(\hat{\mathbf{e}}_{1}, \hat{\mathbf{e}}_{2}\right)\right\}$, where sat $(\cdot)$ denotes the standard saturation function and saturation is applied outside an appropriately defined compact set for the control input $\mathbf{u}$. For details of the stability analysis of the output feedback control design, the reader is referred to [40]. Also note that the forced saturation is applied to the proposed control law if and only if a strictly output feedback controller is used, i.e., if only $\mathbf{e}_{1}$ is available and $\dot{\mathbf{e}}_{1}$ (and consequently $\mathbf{e}_{2}$ ) is immeasurable.

Remark 2: Since HGO is basically an approximate differentiator, thus we can expect that the measurement noise and unmodeled highfrequency sensor dynamics will put a practical limit on how small the observer gain $\epsilon$ could be chosen. On the other hand, the observer gain $\epsilon$ must be chosen as small as possible in order to achieve faster speed of convergence of the observer. Thus, there is a tradeoff between the converging speed of the estimate to the true state and the noise amplification in the presence of noise. Therefore, it is better to avoid use of the estimated $\mathbf{r}(t)$ directly for control implementation, since it would be more sensitive to noise based on the high gain $\alpha_{3} / \epsilon^{3}$ as compared with the much lower gains $\alpha_{1} / \epsilon$ and $\alpha_{2} / \epsilon^{2}$ used to obtain $\hat{\mathbf{e}}_{1}$ and $\hat{\mathbf{e}}_{2}$ as seen in Eq. (39). Finally, we would also like to clarify that the observation of $\hat{\mathbf{r}}$ (even though it is not used directly in the output feedback controller) is necessary to implement the observation of $\hat{\mathbf{e}}_{2}$ (and subsequently $\hat{\mathbf{e}}_{1}$ ) and as can be seen from the second equation in Eq. (39).

\section{Simulation Results}

\section{A. Wing-Section and Disturbance Mode}

In this section, simulation results are presented for a nonlinear 2-DOF aeroelastic system controlled by leading- and trailing- edge flaps and subjected to external disturbances. The nonlinear wingsection model is simulated using the dynamics of Eqs. (1) and (2). The model parameters used in the simulation are the same as used in [21] and listed in Table 1, and a block diagram for the controller is shown in Fig. 2.

Similar to $[\overline{21}, 23]$, the desired trajectory variables $\mathbf{x}_{d}, \dot{\mathbf{x}}_{d}$, and $\ddot{\mathbf{x}}_{d}$ are simply selected as zero. The initial conditions for pitch angle $\alpha(t)$ and plunge displacement $h(t)$ are chosen as $\alpha(0)=5.729 \mathrm{deg}$ and $h(0)=0 \mathrm{~m}$, and all other variables $\dot{h}(t), \dot{\alpha}(t), \ddot{h}(t)$, and $\ddot{\alpha}(t)$ are initially set to zero. The initial parameter estimate $\hat{\theta}(0)$ is set to -2 and $\theta=\bar{U}_{12}=-3.082$, according the model and parameters listed in Table 1. Both the leading-edge $\beta(t)$ and trailing-edge $\gamma(t)$ flaps are constrained to vary from $\pm 15 \mathrm{deg}$. For the numerical example, the

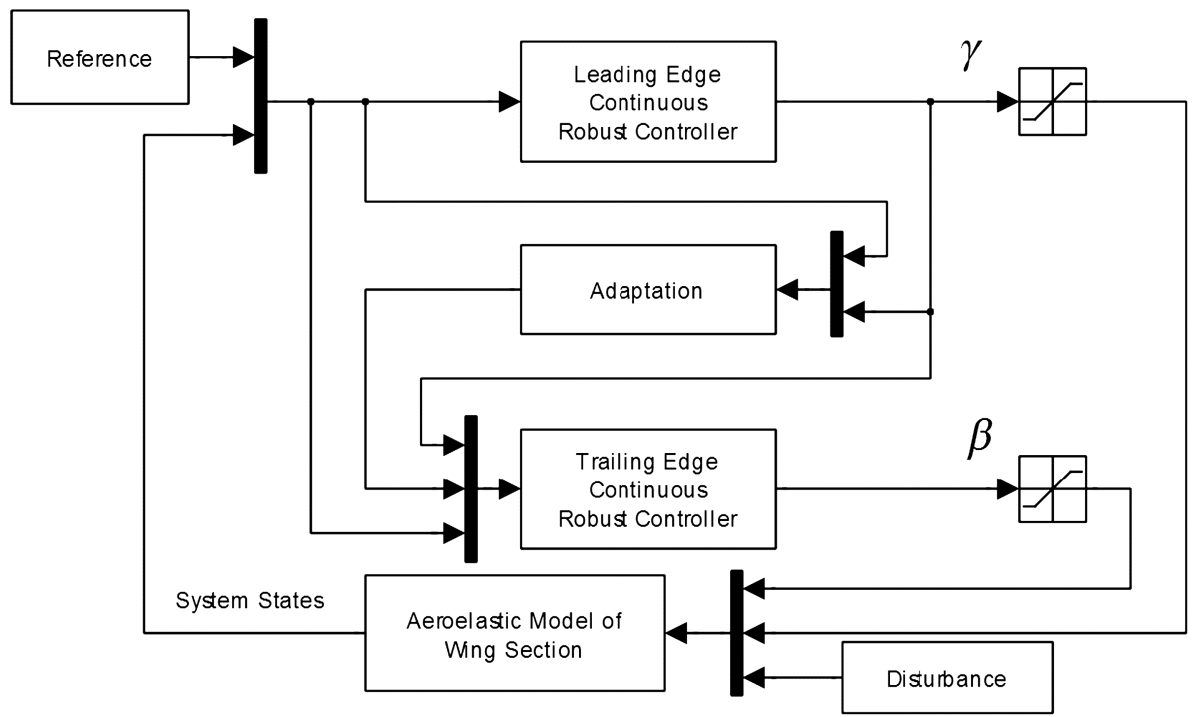

Fig. 2 Controller block diagram. 
Table 2 Simulation parameters

\begin{tabular}{lrrccc}
\hline \hline Freestream velocity & $K_{11}$ & $K_{22}$ & $\Gamma_{11}$ & $\Gamma_{22}$ & $\kappa$ \\
\hline $8 \mathrm{~m} / \mathrm{s}$ & 10 & 10 & 3 & 3 & 10 \\
$13.28 \mathrm{~m} / \mathrm{s}$ & 1 & 1 & 5 & 5 & 10 \\
\hline \hline
\end{tabular}

signs of the leading principal minors of the high-frequency gain matrix $\mathbf{G}_{s}$ are encoded in the diagonal matrix $\mathbf{D}$, and $D_{11}=$ $D_{22}=-1$. In this paper, the following sinusoidal gust is considered as external disturbance:

$$
w_{G}(\tau)=H(\tau) w_{0} \sin \omega \tau
$$

where $\omega=0.5 \mathrm{rad} / \mathrm{s}, w_{0}=0.047 \mathrm{~m} / \mathrm{s}$ in preflutter speed, and $w_{0}=$ $0.07 \mathrm{~m} / \mathrm{s}$ in postflutter speed. Based on our previous work [24], the proposed controller can only compensate the disturbance signal whose magnitude is under certain threshold, since both $\beta(t)$ and $\gamma(t)$ flaps are constrained to vary between $u_{b}= \pm 15 \mathrm{deg}$. Any disturbance larger than that threshold would result in alternate equilibria away from the origin. Hence, in order to show the proposed robust controller can compensator the unknown disturbance, the size of the disturbance can not be arbitrary large.

Another type of external disturbance is modeled as a triangular gust, whose velocity distribution $w_{G}(\tau)$ can be given as
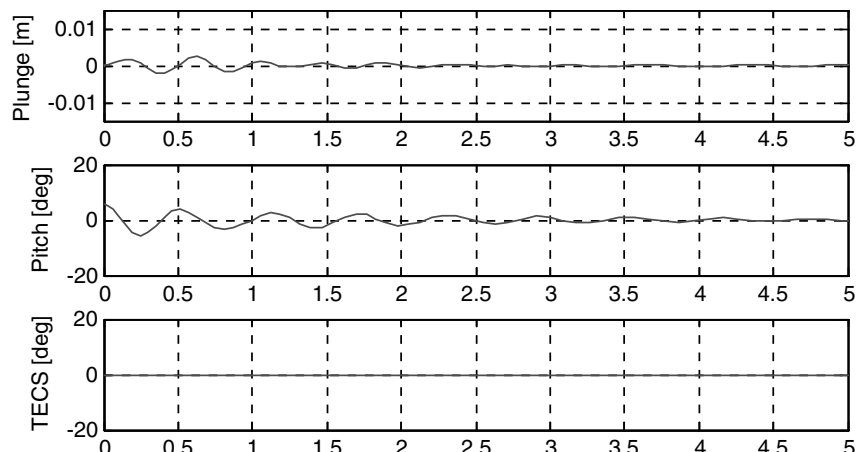

a)

Fig. 3 System response at preflutter speed $U_{\infty}=8 \mathrm{~m} / \mathrm{s}$ : a) open loop and b) closed loop.

\section{B. Controller Implementation} the integrated terms as follows:

where

b)

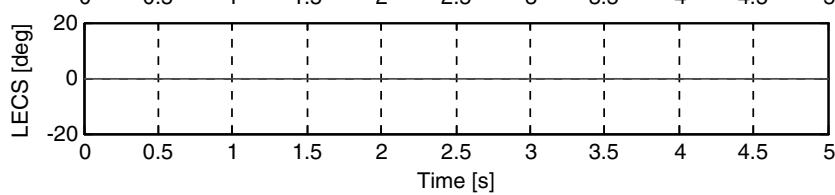

$$
\begin{aligned}
& w_{G}(\tau)=2 w_{0} \frac{\tau}{\tau_{G}}\left(H(\tau)-H\left(\tau-\frac{\tau_{G}}{2}\right)\right) \\
& \quad-2 w_{0}\left(\frac{\tau}{\tau_{G}}-1\right)\left(H\left(\tau-\tau_{G}\right)-H\left(\tau-\frac{\tau_{G}}{2}\right)\right)
\end{aligned}
$$

where $H(\cdot)$ denotes a unit step function and $\tau_{G}=U_{\infty} t_{G} / b$ given $t_{G}=0.25 \mathrm{~s}$. This triangular gust lasts $0.5 \mathrm{~s}$ from $t=0$ to $0.5 \mathrm{~s}$. $w_{0}=0.7 \mathrm{~m} / \mathrm{s}$ in both pre- and postflutter speeds. This type of disturbance is tested in order to demonstrate the performance of the proposed controller under a large ephemeral disturbance that is not $\mathcal{C}^{2}$ smooth and can not be suppressed all the time.

To account for the integral windup problem stemmed from integration of the error signal in the proposed controller and saturation on control signals, a simple antiwindup law is applied to

$$
\dot{\bar{u}}_{i}= \begin{cases}0, & \forall\left|u_{i}\right| \geq u_{b} \quad \text { and } \quad \operatorname{sign}\left(u_{i}\right) \dot{\bar{u}}_{i}>0 \\ \dot{\bar{u}}_{i}, & \text { otherwise }\end{cases}
$$
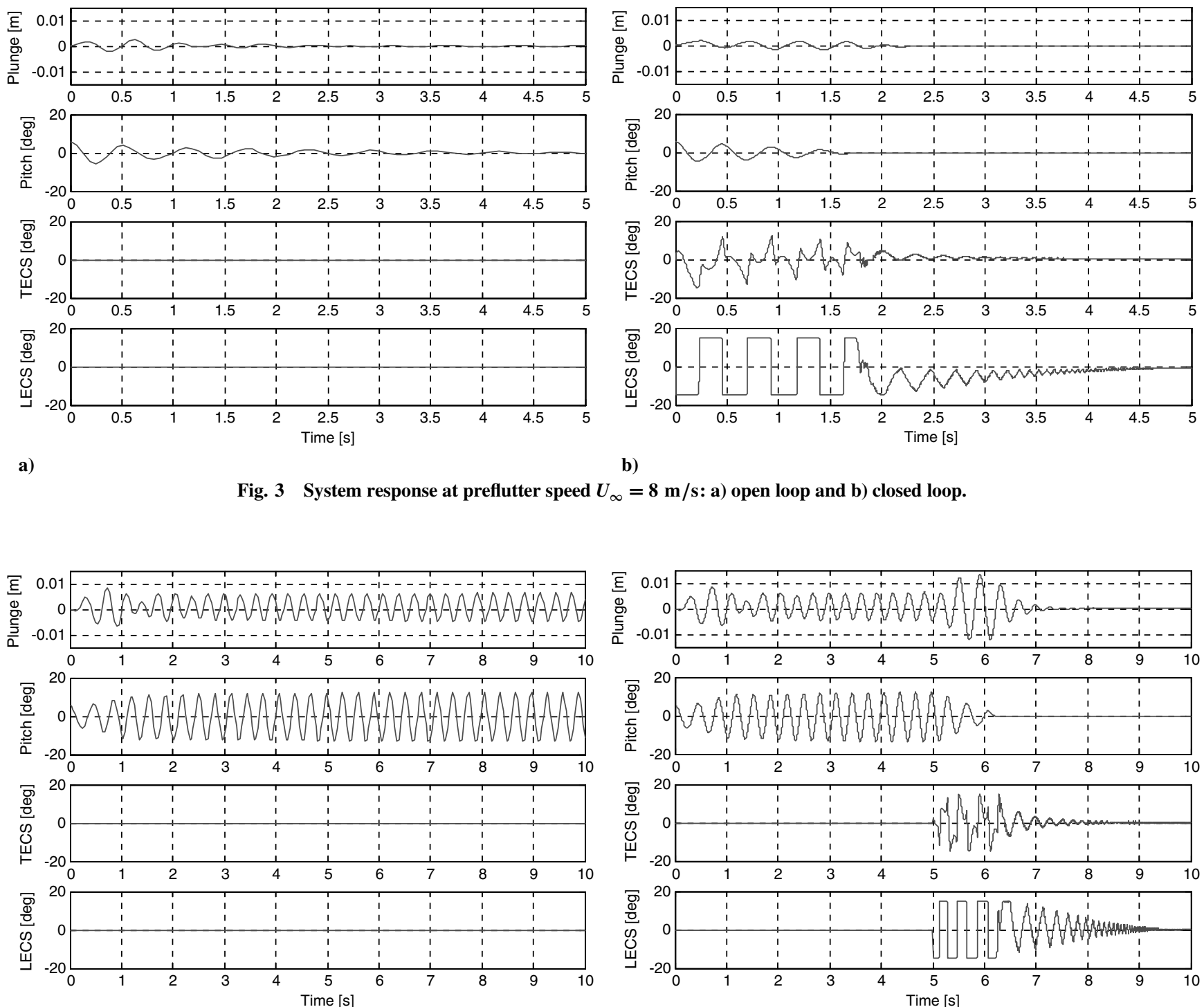

a)
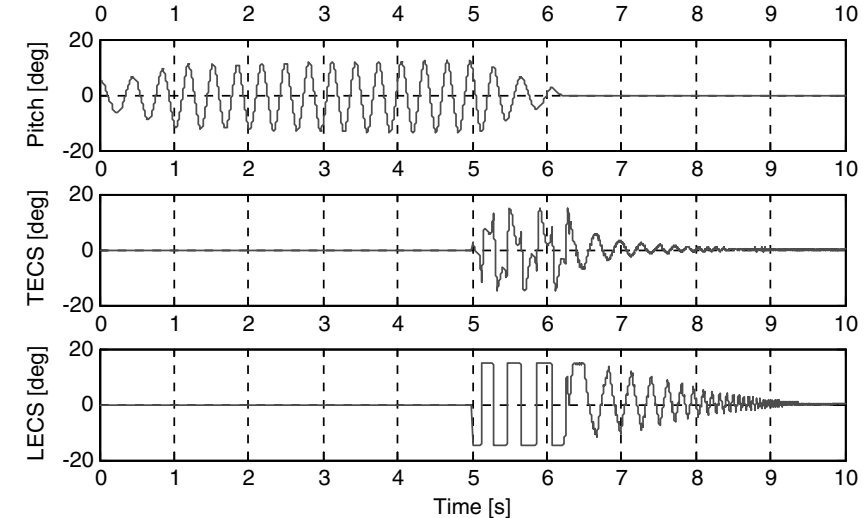

b)

Fig. 4 System response at postflutter speed $U_{\infty}=13.28 \mathrm{~m} / \mathrm{s}$ : a) open loop and b) closed loop. 

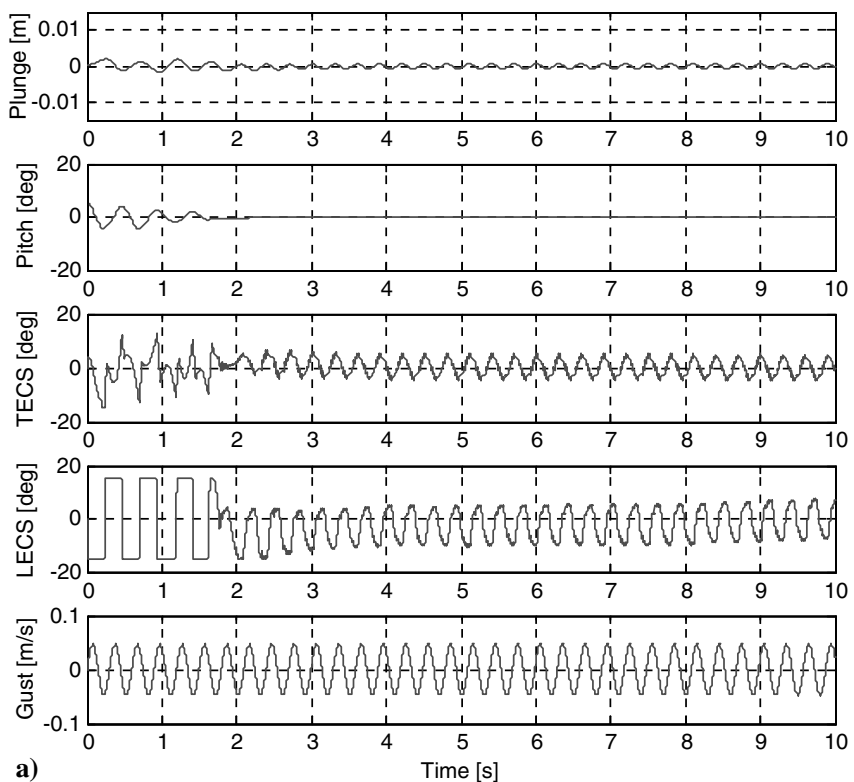

Fig. 5 System response under sinusoidal gust at a) preflutter speed $U_{\infty}=8 \mathrm{~m} / \mathrm{s}$ and b) postflutter speed $U_{\infty}=13.28 \mathrm{~m} / \mathrm{s}$.

$$
\overline{\mathbf{u}}=\mathbf{D}^{-1} \int_{0}^{t}\left[\left[\begin{array}{ll}
Y \hat{\theta} & 0
\end{array}\right]^{T}+(\mathbf{K}+\mathbf{I}) \kappa \mathbf{e}_{2}(\tau)+\Gamma \operatorname{sign}\left(\mathbf{e}_{2}(\tau)\right)\right] \mathrm{d} \tau
$$

and $\bar{u}_{i}$ represents the $i$ th element in $\overline{\mathbf{u}}$. For the parameter adaptation law, based on [24], a method to limit the auxiliary signal $\dot{\hat{\vartheta}}=$ $\Gamma_{Y} Y \kappa e_{21}$ is proposed, according to the magnitude of unsaturated control input signal $\mathbf{u}$, as follows:

$$
\dot{\hat{\vartheta}}_{a}= \begin{cases}\dot{\hat{\vartheta}} g \frac{u_{b}}{\left|u_{1}\right|}, & \left|u_{1}\right|>u_{b} \\ \dot{\hat{\vartheta}} g, & \left|u_{1}\right| \leq u_{b} \quad \text { and } \quad\left|u_{2}\right|>u_{b} \\ \dot{\hat{\vartheta}}, & \left|u_{i}\right| \leq u_{b}, \quad \forall i=1,2\end{cases}
$$

where $\dot{\hat{\vartheta}}_{a}$ denotes the actual auxiliary signal used in Eq. (26). $g$ denotes the auxiliary saturation gains. $u_{i}$ is the $i$ th element in $\mathbf{u}$. The auxiliary saturation gain and adaptation gain are selected as $g=0.1$ and $\Gamma_{Y}=0.01$. The parameters for the controller in pre- and postflutter conditions are listed in Table $\underline{2}$.
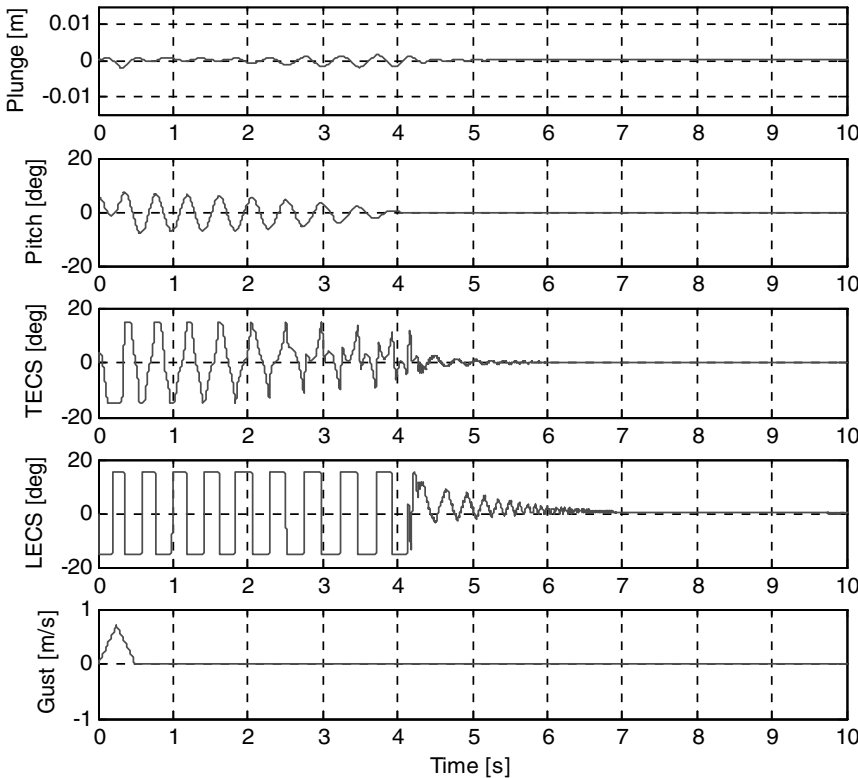

a)

Fig. 6 System response under triangular gust at a) preflutter speed $U_{\infty}=8 \mathrm{~m} / \mathrm{s}$ and b) postflutter speed $U_{\infty}=13.28 \mathrm{~m} / \mathrm{s}$.
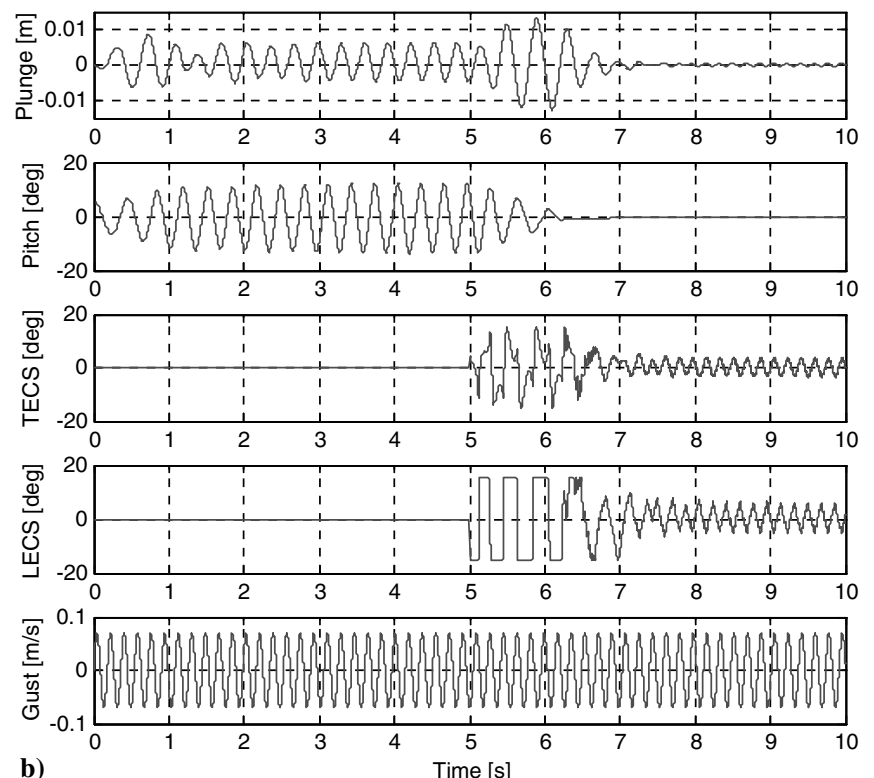

\section{Results}

In this section, simulation results for a 2-D wing-section model under the proposed control are presented. When no external disturbance is considered, Fig. 3 shows the open-loop and closedloop responses of wing-section model at preflutter speed $U_{\infty}=$ $8 \mathrm{~m} / \mathrm{s}<U_{F}=11.4 \mathrm{~m} / \mathrm{s}$. In Fig. $\underline{3 b}$, one can easily see that the proposed control law successfully drives the plunge and pitch displacements to zero within $2 \mathrm{~s}$. Note that the controller is turned on at $t=0[\mathrm{~s}]$. This test was conducted to evaluate the reaction time and control surface displacement characteristics, even if in preflutter speed condition the response will be damped without control actuation. In Fig. $3 b$, the plunge and pitch dynamics are rapidly controlled, within $\overline{2 \mathrm{~s}}$, although the LECS saturates and both flaps will continue operation for about $4.5 \mathrm{~s}$. This phenomena might need to be further investigated as to evaluate the energy expended to operate the control surfaces, and optimization can be carried out to minimize it.

Figure $4 \mathrm{a}$ and $4 \mathrm{~b}$ compare the open-loop and closed-loop responses of the system at postflutter speed $U_{\infty}=13.28 \mathrm{~m} / \mathrm{s}>$ $U_{F}=11.4 \mathrm{~m} / \mathrm{s}$; in the absence of any control input, LCOs are
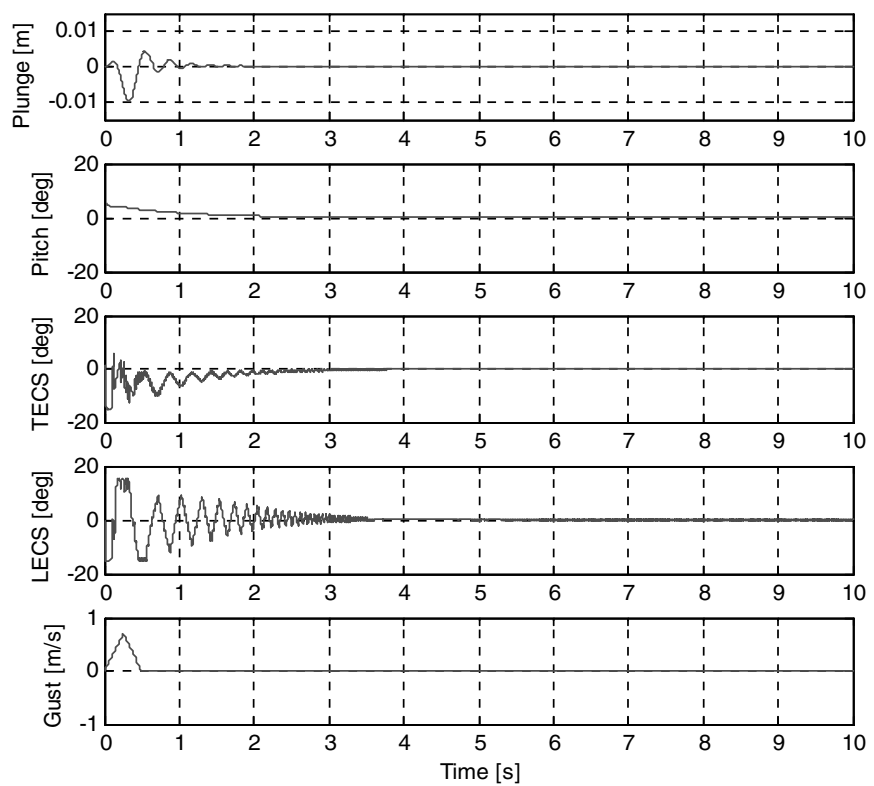

b) 
experienced due to the nonlinearities in the system model. The control is turned on at $t=5 \mathrm{~s}$ in Fig. 4b. As one can see from Fig. 4b, the pitching displacement converges to zero in less than $2 \mathrm{~s}$, whereas it takes a little longer time to suppress the plunging displacement oscillations.

Under a small sinusoidlike gust, with $w_{0}=0.047 \mathrm{~m} / \mathrm{s}$ at both preflutter speed and $w_{0}=0.07 \mathrm{~m} / \mathrm{s}$ at postflutter speed, the closedloop responses of the system are represented in Fig. 5. The control is turned on at $t=0 \mathrm{~s}$ in Fig. $5 \mathrm{a}$ and $t=5 \mathrm{~s}$ in Fig. $5 \mathrm{~b}$. Compared with Figs. $3 \mathrm{~b}$ and $4 \mathrm{~b}$, one can see that it takes nearly the same time $(2 \mathrm{~s})$ for the controller to suppress the pitching and plunging displacements, even in the presence of a sustained external disturbance. One can also clearly see that the control signal is able to compensate for the sinusoidal disturbance injected into the wing-section model.

The closed-loop responses of the system under a large triangular gust $\left(w_{0}=0.7 \mathrm{~m} / \mathrm{s}\right)$ are represented in Fig. 6 at both pre- and postflutter speeds. Note that this triangular gust is not $\mathcal{C}^{2}$ smooth and it can not be compensated all the time under current wing-section model by a limited control signal. From Fig. 6a, one can see that the proposed controller can suppress the pitching and plunging displacements in $4 \mathrm{~s}$ at preflutter speed. It is also clearly to see that it takes less than $3 \mathrm{~s}$ at postflutter speed for the pitching and plunging displacements to converge to zero. Note that in this case, the control is turned on at $t=0 \mathrm{~s}$ at both pre- and postflutter speeds.

\section{Conclusions}

In this paper, a continuous robust feedback controller has been proposed to suppress aeroelastic vibrations on an unmodeled nonlinear plunging and pitching wing section in pre- and postflutter conditions and subjected to external disturbance. The control strategy is implemented via leading-edge $(\gamma)$ and trailing-edge $(\beta)$ control surfaces. By using a Lyapunov-based method for design and analysis, a semiglobal asymptotic stability result on the tracking error is obtained. To account for the integral windup problem generated from the integration of the error signal present in the proposed controller, along with the saturation of the control signals, a simple antiwindup law is implemented. Simulation results under different operating conditions and disturbance loading show the efficacy of the control design.

In future work, an unsteady model based on Theodorsen's function or the corresponding time-domain Wagner indicial function will be considered to describe the aerodynamics with the lag effects. However, the leading and trailing edges with their potential nonlinear flow characteristics that will lead into flow separation and stall might require experimental empirical corrections, and this model is not currently available. Work is in progress to account for dynamic stall and trailing-edge separation using a modified Beddoes-Leishman's formulation. Furthermore, future work will also rigorously consider the effects of actuator saturation and the antiwindup mechanism in the stability analysis.

\section{References}

[1] Mukhopadhyay, V., "Benchmark Active Control Technology: Part 1," Journal of Guidance, Control, and Dynamics, Vol. 23, No. 5, 2000, pp. 913-960. doi: $10.2514 / 2.4631$

[2] Mukhopadhyay, V., "Benchmark Active Control Technology: Part 2," Journal of Guidance, Control, and Dynamics, Vol. 23, No. 6, 2000, pp. 1093-1139. doi: $10.2514 / 2.4659$

[3] Mukhopadhyay, V., "Benchmark Active Control Technology: Part 3," Journal of Guidance, Control, and Dynamics, Vol. 24, No. 1, 2001, pp. 146-192. doi: $10.2514 / 2.4693$

[4] Friedmann, P. P., Guillot, D., and Presente, E., "Adaptive Control of Aeroelastic Instabilities in Transonic Flow and Its Scaling," Journal of Guidance, Control, and Dynamics, Vol. 20, No. 6, 1997, pp. 11901199 doi: $10.2514 / 2.4175$

[5] Wang, Z., Behal, A., and Marzocca, P., "Advances in the Adaptive Robust Control of Aeroelastic Structural Systems," International
Journal of Aeronautical and Space Sciences, Vol. 11, No. 4, 2010, pp. 285-302 doi:10.5139/IJASS.2010.11.4.285

[6] Librescu, L., and Marzocca, P., "Advances in the Linear/Nonlinear Control of Aeroelastic Structural Systems," Acta Mechanica, Vol. 178, No. 3-4, Aug. 2005, pp. 147-186. doi:10.1007/s00707-005-0222-6

[7] Lind, R., and Brenner, M., Robust Aeroservoelastic Stability Analysis, Springer-Verlag, New York, 1999.

[8] Barker, J. M., and Balas, G. J., "Comparing Linear Parameter-Varying Gain-Scheduled Control Techniques for Active Flutter Suppression," Journal of Guidance, Control, and Dynamics, Vol. 23, No. 5, 2000 pp. 948-955. doi: $10.2514 / 2.4637$

[9] Scott, R. C., and Pado, L. E., "Active Control of Wind-Tunnel Model Aeroelastic Response Using Neural Networks," Journal of Guidance, Control, and Dynamics, Vol. 23, No. 6, 2000, pp. 1100-1108. doi: $10.2514 / 2.4661$

[10] Zhang, R., and Singh, S. N., "Adaptive Output Feedback Control of an Aeroelastic System with Unstructured Uncertainties," Journal of Guidance, Control, and Dynamics, Vol. 24, No. 3, 2001, pp. 502-509. doi: $10.2514 / 2.4739$

[11] Zeng, Y., and Singh, S. N., "Output Feedback Variable Structure Adaptive Control of Aeroelastic Systems," Journal of Guidance, Control, and Dynamics, Vol. 21, No. 6, 1998, pp. 830-837. doi: $10.2514 / 2.4342$

[12] Xing, W., and Singh, S. N., "Adaptive Output Feedback Control of a Nonlinear Aeroelastic Structure," Journal of Guidance, Control, and Dynamics, Vol. 23, No. 6, 2000, pp. 1109-1116. doi: $10.2514 / 2.4662$

[13] Behal, A., Rao, V. M., Marzocca, P., and Kamaludeen, M., "Adaptive Control for a Nonlinear Wing Section with Multiple Flaps," Journal of Guidance, Control, and Dynamics, Vol. 29, No. 3, 2006, pp. 744-749. doi: $10.2514 / 1.18182$

[14] Rao, V. M., Behal, A., Marzocca, P., and Rubillo, C. M., "Adaptive Aeroelastic Vibration Suppression of a Supersonic Airfoil with Flap," Aerospace Science and Technology, Vol. 10, 2006, pp. 309-315. doi:10.1016/j.ast.2006.03.006

[15] Lazarus, K., "Multivariable High-Authority Control of Plate-Like Active Lifting Surfaces," Ph.D. Dissertation, Department of Aeronautics and Astronautics, Massachusetts Inst. of Technology, Cambridge, MA, June 1992.

[16] Singh, S. N., and Wang, L., "Output Feedback Form and Adaptive Stabilization of a Nonlinear Aeroelastic System," Journal of Guidance, Control, and Dynamics, Vol. 25, No. 4, 2002, pp. 725-732. doi:10.2514/2.4939

[17] Behal, A., Marzocca, P., Rao, V. M., and Gnann, A., "Nonlinear Adaptive Control of an Aeroelastic Two-Dimensional Lifting Surface," Journal of Guidance, Control, and Dynamics, Vol. 29, No. 2, 2006, pp. 382-390. doi:10.2514/1.14011

[18] Lee, K. W., and Singh, S. N., "Global Robust Control of an Aeroelastic System Using Output Feedback," Journal of Guidance, Control, and Dynamics, Vol. 30, No. 1, 2007, pp. 271-275. doi: $10.2514 / 1.22940$

[19] Zhang, F., and Soffker, D., "Active Flutter Suppression of a Nonlinear Aeroelastic System Using PI-Observer," Motion and Vibration Control, edited by H. Ulbrich, and L. Ginzinger, Springer, Amsterdam, 2009, pp. 367-376.

[20] Lee, K. W., and Singh, S. N., "Immersion and Invariance Based Adaptive Control of a Nonlinear Aeroelastic System," Journal of Guidance, Control, and Dynamics, Vol. 32, No. 4, 2009, pp. $1100-1110$ doi: $10.2514 / 1.42475$

[21] Platanitis, G., and Strganac, T. W., "Control of a Nonlinear Wing Section Using Leading- and Trailing-Edge Surfaces," Journal of Guidance, Control, and Dynamics, Vol. 27, No. 1, 2004, pp. 52-58. doi:10.2514/1.9284

[22] Gujjula, S., Singh, S. N., and Yim, W., "Adaptive and Neural Control of a Wing Section Using Leading- and Trailing-Edge Surfaces," Aerospace Science and Technology, Vol. 9, 2005, pp. 161-171. doi:10.1016/j.ast.2004.10.003

[23] Reddy, K. K., Chen, J., Behal, A., and Marzocca, P., "Multi-Input/ Multi-Output Adaptive Output Feedback Control Design for Aeroelastic Vibration Suppression," Journal of Guidance, Control, and Dynamics, Vol. 30, No. 4, 2007, pp. 1040-1048. doi: $10.2514 / 1.27684$

[24] Wang, Z., Behal, A., and Marzocca, P., "Model-Free Control Design for MIMO Aeroelastic System Subject to External Disturbance," 
Journal of Guidance, Control, and Dynamics, Vol. 34, No. 2, 2011, pp. 446-458.

doi: $10.2514 / 1.51403$

[25] Sastry, S., and Bodson, M., Adaptive Control: Stability, Convergence, and Robustness, Prentice-Hall, Upper Saddle River, NJ, 1989.

[26] Ioannou, P., and Sun, K., Robust Adaptive Control, Prentice-Hall, Englewood Cliffs, NJ, 1996.

[27] Krstic, M., Kanellakopoulos, I., and Kokotovic, P., Nonlinear and Adaptive Control Design, Wiley, New York, 1995.

[28] Chen, J., Behal, A., and Dawson, D. M., "Adaptive Output Feedback Control for a Class of MIMO Nonlinear Systems," Proceedings of the American Control Conference, Minneapolis, MN, 2006 pp. 5300-5305.

[29] Wang, Z., Chen, J., and Behal, A., "Robust Adaptive Control Design for a Class of Uncertain MIMO Nonlinear Systems," Proceedings of the IEEE Multi-Conference on Systems and Control, Yokohama, Japan, 2010, pp. 2284-2289.

[30] Zhang, X., Behal, A., Dawson, D. M., and Xian, B., "Output Feedback Control for a Class of Uncertain MIMO Nonlinear Systems with NonSymmetric Input Gain Matrix," Proceedings of the IEEE Conference on Decision and Control, Seville, Spain, 2005, pp. 7762-7767.

[31] Chen, J., Behal, A., and Dawson, D. M., "Robust Feedback Control for a Class of Uncertain MIMO Nonlinear Systems," IEEE Transactions on Automatic Control, Vol. 53, No. 2, March 2008, pp. 591-596. doi:10.1109/TAC.2008.916658

[32] Lewis, F. L., Campos, J., and Selmic, R., Neuro-Fuzzy Control of Industrial Systems with Actuator Nonlinearities, Society for Industrial and Applied Mathematics, Philadelphia, 2002.
[33] Utkin, V. I., Sliding Modes in Control and Optimization, SpringerVerlag, New York, 1992.

[34] Xian, B., Dawson, D. M., de Queiroz, M. S., and Chen, J., "A Continuous Asymptotic Tracking Control Strategy for Uncertain Nonlinear Systems," IEEE Transactions on Automatic Control, Vol. 49, No. 7, July 2004, pp. 1206-1211. doi:10.1109/TAC.2004.831148

[35] Marzocca, P., Librescu, L., and Chiocchia, G., "Aeroelastic Response of 2-D Lifting Surfaces to Gust and Arbitrary Explosive Loading Signatures," International Journal of Impact Engineering, Vol. 25, 2001, pp. 41-65. doi:10.1016/S0734-743X(00)00033-6

[36] Leishman, J. G., and Beddoes, T. S., "A Semi-Empirical Model for Dynamic Stall," Journal of the American Helicopter Society, Vol. 34, No. 3, 1989, pp. 3-17.

[37] Hansen, M. H., Gaunaa, M., and Madsen, H. A., "A BeddoesLeishman Type Dynamic Stall Model in State-space and Indicial Formulations," Ris $\emptyset$ National Lab., Ris $\varnothing-R-1354(E N)$, Roskilde, Denmark, 2004.

[38] Arena, A., Lacarbonara, W., and Marzocca, P., "Nonlinear Aeroelastic Formulation for Flexible High-Aspect Ratio Wings via Geometrically Exact Approach," AIAA Paper 2011-1916, Denver, CO, April 472011.

[39] Morse, A. S., "A Gain Matrix Decomposition and Some of its Applications," Systems and Control Letters, Vol. 21, 1993, pp. 1-10. doi:10.1016/0167-6911(93)90038-8

[40] Khalil, H., Nonlinear Systems, Prentice-Hall, Upper Saddle River, NJ, 1996. 